\title{
Benefits of area-wide traffic calming measures: outcomes still to assess
}

\author{
A. Granà ${ }^{1}$, T. Giuffrè ${ }^{2} \&$ M. Guerrieri ${ }^{1}$ \\ ${ }^{I}$ Department of Road Infrastructure Engineering, \\ Palermo University, Italy \\ ${ }^{2}$ Department of Aeronautics Technologies and Infrastructures, \\ Palermo University, Italy
}

\begin{abstract}
The role of traffic calming schemes in urban network management is mainly to minimize the undesirable effect of traffic in residential areas; in this context accident reduction can be a realistic objective. Several studies, indeed, inform us that traffic calming is a major part of the treatments available to reduce road accidents in urban areas. Paradoxically the increase of the accident rate per kilometre travelled has been observed in the urban context as result of the wellknown accident migration phenomenon. In connection to these considerations, the effectiveness of traffic calming measures on road safety is discussed in this paper. Then, after a synthetic exposition of the conceptual formulation and the potential of the meta-analysis method in detecting the true safety effect of traffic calming measures, the paper also considers the role of road network planning and the characteristics of the urban network that have to be consistent with the traffic calming objectives. Lastly, a methodological framework to follow for the implementation of traffic calming schemes in residential areas, from the planning level to the road design level, is proposed.
\end{abstract}

Keywords: road safety, traffic calming scheme, meta-analysis.

\section{Introduction}

Strategies targeted to improve road safety follow in general two different aims: i) reducing the severity of the consequences of risky behaviours by the installation of devices useful to contain driver and passenger damage when collision occurs; ii) making the consequences of risky behaviour more severe by the installation of 
suitable measures alerting users and inducing them to conventional behaviours. Traffic calming devices are referable to the last one aim; acting on driver behaviours they can paradoxically increase the accident rate per km travelled [1]. Moreover, undesirable safety effects of traffic calming schemes are empirically explained by the traffic volume shift from the treated road sections to other roads. In fact, an in-depth analysis of the effects of different schemes and treatments on drivers' speed highlights that the benefits are generally localized in time and space, but other inconveniences (i.e. the lack of public acceptability, noise and air pollution, possible accident migration) can occur together. Many scientific literature reports on this matter also agree on the risk related to episodic traffic calming installations, or to schemes limited at isolated sites, and to the absence of the knowledge of the area-wide effects that traffic calming measures can produce. This paper, on the basis of an oriented literature review, aims to perceive the area-wide effects of traffic calming measures and to consider their role in road network planning. Lastly, a methodological framework to follow for the implementation of traffic calming schemes in residential areas, from the planning level to the road design level, is proposed.

\section{Effects of traffic calming measures on road safety}

In residential streets (both on access roads and on the local-distributor network) accidents are not concentrated in black spots, but scattered over larger areas. So specific localized countermeasures can be unsuited for contrasting such accident patterns and a global approach can be more effective. From the safety point of view, the implementation of traffic calming measures in urban residential areas is based on the assumption that the speed reduction following from traffic calming installations achieves an effective reduction both of crash severity and of accident occurrence, but such safety improvement (expressed in terms of accident reductions) can be greatly variable depending on environmental and traffic factors defining the context of the application (class of road, dimension of the interested area, type of adopted measures, changes in traffic demand). Literature on traffic calming safety evaluation includes a great variety of studies, differing for year of publication, country of origin, study design and controlled parameters; further differences concern accident severity and type of road to which results refer; an exhaustive retrieval of such studies up to 1997 is reported by Elvik et al. [2] and Elvik [3]. More recent studies on this subject are referred in [4-7].

Despite the rich harvest of information one can draw from reports by safety research institutes or journals and conference proceedings, in the field of traffic calming safety evaluation we have to pay for the generally poor reliability of the results, most of the time carried out from methodological approaches quite lacking in scientific foundations. From a methodological point of view it has to be observed that the analysis of the accident phenomenon, also in the case of the safety evaluation of traffic calming measures, is not an exact science, but it is founded above all on the observation of accidents by empirical approaches, as before-and-after studies; misleading conclusions can essentially derive either 
from lack of control for regression-to-the-mean (or long-term trends in accident occurrence) or by ignoring the presence of potentially important confounding factors. An example of relevant bias due to regression-to-the-mean can occur in the safety evaluation of major (access) roads with high accident numbers in the before period; lack of control for confounding factors (change in traffic volume, modifications in land use, etc.) can entirely compromise the area wide safety evaluation. As is well known the way to prevent both kinds of bias has been codified by Hauer [8] by means of the Empirical-Bayesian approach to the before and after observational studies, but up until now no application to traffic calming safety evaluation meeting this standard has been carried out. Only a few studies employ a matched comparison group and in this way they implicitly control the regression-to-the-mean bias; particularly if the matched comparison area has an equally bad or good accident record as the traffic calming area in the before period [3].

In the absence of EB before-and-after or other kinds of sound studies on which traffic calming safety evaluation could be based, an approach by metaanalysis can still provide an effective tool to review literature results and to draw out realistic estimates of traffic calming safety effects; it of course cannot remove biased results from source studies, but by means of subjective judgement, it allows one to establish a hierarchy of values between them on the basis of their objective credit. In this way meta-analysis approach enables one to gather and to analyze contributions from different studies starting from the evaluation and the interpretation of their methodological design. It has to be noted that a meta-analysis procedure has to provide for checking the initial hypothesis and the robustness of conclusions in the source studies design. At the same time it has to control a particular bias specially arising in literature retrieval, known as "publication bias". This can occur either when results are not statistically significant or when they are regarded as 'unfavourable' or 'negative' (increase in number or severity of accidents); in both cases results are less likely to be published than statistically significant or "desirable" ones. A critical synthesis of the main results of meta-analysis studies applied to traffic calming schemes will be given in the following, but it will be preceded by an overview of the conceptual formulation of the method.

\section{An overview of the meta-analysis}

Meta-analysis is a statistical method that in lots of scientific research sectors is the basis of analysis procedures directed towards the effects evaluation of ameliorative measures. So the meta-analysis collecting and examining data from different studies on a specific theme can be applied to identify the common effect of a treatment, when this (or the effect size) is consistent from one study to the next; on the contrary, when the effect size is not exactly the same in all the studies, the meta-analysis can be applied to explain the above-mentioned variation. The reasons for the meta-analysis application are referable to the decision on the usefulness of an ameliorative measure, or on the validity of a hypothesis; this decision cannot be based on conclusions of a single study, 
because the results can vary from one study to another. The meta-analysis, on the contrary, makes use of suitable formulations for elaborating data from more researches (similar to the elaborations of a single study), whatever the number may be. It is directed towards the quantitative estimate of the global effect (or the combined effect) of a specific treatment (or a specific variable); but rather than compute a simple mean of the effect sizes, the meta-analysis computes a weighted mean of the effect sizes, with more weight given to some studies and less weight given to others. The models used in meta-analysis are the fixed effect model and the random effects model [9]. The first, starting from analogous studies (as regards the modes of the sample selection and/or of the execution of tests) allows one to deduce the common effect of a treatment (or the effect on average); the second one, starting from data collected by different studies on the same theme (i.e. on the effect of a treatment) and from the evaluation of differences among the studies, allows one to evaluate if the afore-mentioned differences could be directed towards (or not) a reliable result on the effect of an ameliorative measure. The random effects model is more generalizable than the fixed affect one, based on an identical and narrowly defined population.

\subsection{Fixed effect model}

The fixed effect model assumes only one true effect size, exactly alike in all the analyzed studies; so the combined effect is the estimate of the common effect size. The only reason for variation is imputable to the random error inherent in each study. When assigning weights to the different studies, small studies can be ignored, because information about the same effect size can be obtained by studies with a wider sample base. The observed effects are thought to be distributed with mean $\mu$, equal in all the studies, and a variance $\sigma^{2}$ depending on the sample size for each study. Therefore, the observed effect is equal to $T_{i}=\mu+\varepsilon_{i}$, where $\varepsilon_{i}$ is the within-study error, i.e. the error correlated to random factors intervened in the sampling within the same population (see Figure 1).

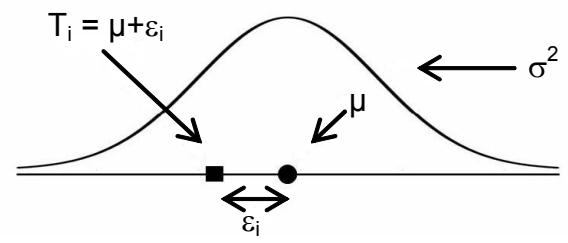

Figure 1: $\quad$ Fixed effect model.

This error decreases when the sample size increases. To minimize the variance of the combined effect, the weight assigned to each study is equal to the inverse of the variance; the inverse variance, roughly proportional to sample size, is computed as:

$$
\mathrm{w}_{\mathrm{i}}=1 / v_{i}
$$


where $\mathrm{v}_{i}$ is the within-study variance for the study $(i)$. The weighted mean $\left(\overline{\mathrm{T}_{0}}\right)$ for the observed effects is then:

$$
\overline{T_{0}}=\left(\sum_{i=1}^{k} w_{i} T_{i}\right) /\left(\sum_{i=1}^{k} w_{i}\right)
$$

The variance of the combined effect is defined as the reciprocal of the sum of the weights:

$$
v_{0}=1 / \sum_{i=1}^{k} w_{i}
$$

The limits of the confidence interval containing $\mu$ at an assigned probability level can then computed in the usual way.

\subsection{Random effect model}

The random effect model assumes that the true effect can change from one study to the next. The examined studies are a random sample of the distribution of true effects; so the combined effect is the mean effect of this distribution.

Studies with a large sample size can offer more accurate estimates than small studies, but each study estimates a different effect value; moreover each estimate represents a sample of the population of which the mean would be estimated. Weights assigned under random effects will be more balanced than those assigned under fixed effects, because the random effect model does not give extreme emphasis to studies with a very large sample size or it does not cancel studies characterized by a small sample size. Nevertheless, two sampling levels and two error levels can be considered as possible: i) each study is used to estimate the true effect in a specific sample population; ii) all the true effects are then used to estimate the mean of the true effects. As a consequence the estimate of the combined effect depends on the number of elements in each study (the first source of error) and on the total number of studies (the second source of error). In other words, even if each study has a wide sample base, the estimate of the mean can be not-exhaustive, because the studies have been extracted by all the possible studies. The observed effect $T_{i}$, given $\theta_{i}$, is sampled from a distribution with mean $\theta_{i}$ and variance $\sigma^{2}$, depending on the study sample size. Then the true effect $\theta_{i}$, in turn, is sampled from a distribution with mean $\mu$ and variance $\tau^{2}$ (see Figure 2). The observed effect is equal to:

$$
T_{i}=\theta_{i}+\varepsilon_{i}=\mu+\zeta_{\mathrm{i}}+\varepsilon_{I}
$$

where $\varepsilon_{i}$ is the within-studies error and $\zeta_{i}$ is the error between studies. In order to consider the tow source of error, the random effect analysis considers primarily the decomposition of the observed variance into two components (within-studies and between-studies) and then the use of both parts in assigning weights. Similarly to the fixed effect model, the random effect model also considers that the weight assigned to each study is equal to the inverse of the variance, with the difference that in this case the variance is composed of two parts. 


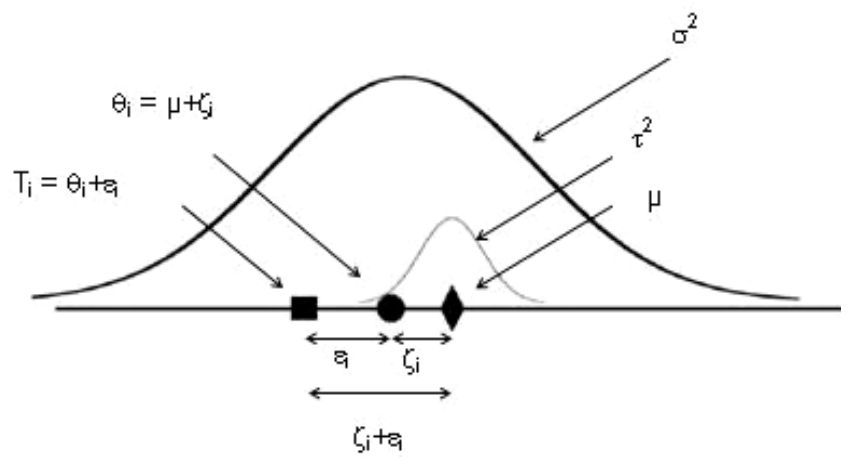

Figure 2: $\quad$ Random effect model.

\subsection{An application of meta-analysis as a tool to evaluate traffic calming effects}

According to Elvik [3], the results of a meta-analysis from 33 studies carried out in 8 different countries on traffic calming effects showed that these measures allow a reduction of injury accidents. In particular, this reduction is higher for residential streets than for main roads. Damage-only accidents are also interested by similar reductions. Measures that have been included in the meta-analysis varied with regard to the surrounding context (mainly with a residential character and a size ranging from $0,25 \mathrm{~km}^{2}$ up to $1,5 \mathrm{~km}^{2}$ ) and to the main traffic function of the road. The reliability of the studies to examine has been valued basing on: i) study organization and applied method; ii) traffic volume data; iii) accident severity; iv) effects on road safety by type of road. The research included beforeand-after studies on accidents occurred in presence of traffic calming measures, but no study controlled explicitly for the regression-to-the-mean or temporal trends in the long period. Table 1 shows the variations in the number of accidents distinguished by severity and by type of road.

Table 1: $\quad$ Results by accident severity and type of road.

\begin{tabular}{|c|c|c|c|c|c|}
\hline \multirow{3}{*}{ Accident severity } & \multirow{2}{*}{ type of road } & \multicolumn{3}{|c|}{ Percentage variation in accidents } \\
\cline { 3 - 6 } & & fixed effect model & \multicolumn{2}{c|}{ random effect model } \\
\cline { 3 - 6 } & estimate & $95 \%$ c.i. $*$ & estimate & $95 \%$ c.i. $*$ \\
\hline \multirow{3}{*}{ injury accidents } & area-wide & -15 & $(-17 ;-12)$ & -15 & $(-19 ;-10)$ \\
\cline { 2 - 6 } & main roads & -8 & $(-12 ;-5)$ & -8 & $(-13 ;-2)$ \\
\cline { 2 - 6 } & local roads & -24 & $(-28 ;-18)$ & -34 & $(-43 ;-23)$ \\
\hline \multirow{3}{*}{ damage-only accidents } & area-wide & -16 & $(-19 ;-13)$ & -19 & $(-26 ;-12)$ \\
\cline { 2 - 6 } & main roads & -11 & $(-16 ;-6)$ & -18 & $(-31 ;-3)$ \\
\cline { 2 - 6 } & local roads & -29 & $(-25 ;-22)$ & -42 & $(-54 ;-26)$ \\
\hline \multirow{3}{*}{ No data on severity } & area-wide & -19 & $(-25 ;-12)$ & -18 & $(-27 ;-9)$ \\
\cline { 2 - 6 } & main roads & -14 & $(-21 ;-6)$ & -13 & $(-21 ;-4)$ \\
\cline { 2 - 6 } & local roads & -57 & $(-68 ;-43)$ & -57 & $(-68 ;-43)$ \\
\hline
\end{tabular}

* The $95 \%$ confidence interval. 
The results related both to the fixed effect model and to the random effect model seem similar and they result significant by a statistical point of view at a $5 \%$ confidence level. The confidence intervals are much wider for the random effect model. This can be explained regarding that the fixed effect model considers only the random variation of effects in each study; on the contrary, the random effect model captures the systematic variation of effects among the examined studies. The reduction of accidents number ranged from 15 per cent to 20 per cent in residential areas where traffic calming scheme were installed. Reductions on local roads ranged from 25 per cent to 55 per cent, whereas reductions found on main roads ranged from 8 per cent to 15 per cent. Results are reasonable because traffic calming measures can reduce traffic volumes and speeds on local roads; on the contrary, these measures can cause an increase in traffic volumes on main roads.

Another meta-analysis literature review from 16 controlled before-and-after studies was conducted by Bunn et al. [10] by means of a random effect model. It was found that area-wide traffic calming in towns and cities has the potential to reduce road traffic injuries (up to $11 \%$ for fatal and non-fatal), but no reliable evidence in reducing the number of road traffic accidents was highlighted. This is not inconsistent with a reduction in the occurrence of injury, since traffic calming primarily reduce vehicle speeds. Authors observe that several methodological issues may have influenced their results and they explicitly warn for the significant heterogeneity between the studies reporting the number of road traffic injuries and accidents.

In order to underline the significance of results derived from the metaanalysis (i.e. the combined effect and the variance), it can be useful to establish a comparison with results derived from only one smaller study.

As example of this, we will refer to Brilon and Blanke [11] report on the effects of area-wide traffic calming measures in six German cities; on the basis of this report we would have to wait for: i) 63 per cent reduction, on average, of seriously injured persons in traffic calmed areas; ii) 50 per cent reduction, on average, of injury accidents; iii) 23 per cent reduction of injury accidents in the studied areas as a whole (including urban arterials and sites without traffic calming measures).

It can be deduced that the meta-analysis including more observations than only one case study, as well as considering lots of factors that can influence the phenomenon under examination, is able to produce more realistic evaluations (closer to the true effect) of safety benefits of traffic calming measures.

\section{Road network planning related to traffic calming measures}

Traffic calming installations in a specific area has to be founded on a road network planning in which the presence of sectors with a strong residential connotation is already recognized; moreover the main aims of the network planning including traffic calming treatments have to count that vehicular traffic move away from the residential areas. This suggests that road network is structured in order to avoid that roads with traffic function are within the same 
area in which the main function is the local one. The road network structure has to be characterized by destinations within the same area attainable through the possible shorter distance. Moreover the existence of infrastructures for alternative transport modes has to be verified to cover the possible removal of road space to motorized traffic.

\subsection{Effects of the area size}

The road classification into functional classes (not being only a simple attribution of possible functions to each class) makes clear a link between the road geometric design and its use; this link represents a safety prerequisite.

The introduction at a network level of traffic calming schemes requests one to distinguish the residential function from the traffic one. The first is suitable for roads allowing the access to properties at margins or for roads characterized by a strong commercial connotation. The second belongs to distribution roads that are often characterized by high traffic volume at relatively high speeds. For safety reasons and in order to increase the residential function of a road by the implementation of traffic calming measures an analogous function has to be assigned to more next roads to tackle altogether them as a residential street. The positive effects from a safety point of view generally increase with the size of the treated area. This is referable to the reduction of the less safe paths within the area, as well as the reduction of the number of intersections with main roads, being reduced the needs to cross them. Van Minnen (1999), as referred by [6], asserts that there is an upper limit for the size of residential areas. This upper limit has to be set by safety, liveability and accessibility criteria: i) if the size exceeds 100 hectare, traffic volumes on the surrounding roads become too large; ii) if the size exceeds 200 hectare, traffic volumes on residential streets can become high, too. Table 2 shows the effects of the size of a residential area on different (quantitative and qualitative) parameters characterizing each of the above-mentioned criteria.

Table 2: $\quad$ Criteria to determine the maximum size of a residential area for traffic calming measure installations [6].

\begin{tabular}{|l|l|}
\hline objectives & General and specific criteria \\
\hline & Limit the travelled distance within the area (residential and distributor roads) \\
Limit the traffic volumes within the area \\
Prevent through-going motorized traffic through the area \\
Limit speeds of motorized traffic
\end{tabular}




\subsection{Effects of the network structure on traffic volumes and road safety}

The structure of road network and the number of connections with the higher order distributor roads determine the volume of motorized traffic within the residential area. From a road safety point of view and for environmental reasons through-traffic within the residential area would have to be excluded; traffic volumes in residential areas would need to be as low as possible and they would enter the residential area without large detours. Table 3 summarizes the most relevant characteristics of three network structures for residential areas [6].

Table 3: $\quad$ Basic residential area network structures [6].

\begin{tabular}{|c|c|c|c|}
\hline \multirow{3}{*}{$\begin{array}{l}\text { The four most relevant } \\
\text { indicators. }\end{array}$} & \multicolumn{3}{|c|}{ Types of residential area network structures } \\
\hline & & & \\
\hline & Grid network & $\begin{array}{l}\text { Limited access } \\
\text { network }\end{array}$ & $\begin{array}{l}\text { Organic } \\
\text { network }\end{array}$ \\
\hline Avoidance of through traffic & - & + & ++ \\
\hline $\begin{array}{l}\text { Short distances for } \\
\text { destination traffic }\end{array}$ & ++ & + & - \\
\hline Self-induced speed reduction & - & + & ++ \\
\hline $\begin{array}{l}\text { Limited number of } \\
\text { connections with distributor } \\
\text { roads }\end{array}$ & - & + & ++ \\
\hline
\end{tabular}

In particular, organic network structures result more consistent to traffic calming criteria than the other two network types: it is best in discouraging through-traffic in residential streets and has the highest safety standard by nature (in fact, they have a large share of T-intersections, which are safer than $\mathrm{X}$ intersections). A point of concern is the central street of the network, which has to carry a relative large amount of approaching traffic (i.e. the origin/destination traffic from and toward the area) and as such it may easily turn into an internal barrier for residents. A solution can be found in increasing the number of nonmotorized urban trips at a loss of the number of motorized urban trips. The number of connections between the residential area and the surrounding distributor roads is a characteristic of the network structure which has effects on the distance travelled inside the residential area and the distance travelled on the distributor roads. The analysis of this matter [6] allowed one to deduce that the higher the number of connections, the smaller the distance travelled both on residential streets and on the surrounding distributor traffic. 


\section{A methodological procedure for implementing a traffic calming zone}

Traffic calming measures can represent a tool for integrating traffic components, specially cars and pedestrians. By this point of view, it can be seen as opposite of approaches accentuating the physic division among mobility components, as a guarantee of road safety. The division by type of mobility represents a systematic practice suitable for engendering a zoned system within road networks scarcely permeable and not much devoted to exchange; nevertheless, qualitative features and the availability of urban spaces can result negatively affected: vehicles tend to appropriate exclusively the road, as well as aggressive behaviour are led to non-motorized traffic, especially near to the greatest points of conflict. Integrating the transport modes points of conflicts increase, but they are characterized by a slower speed and by less aggressive behaviours; this allows a higher level of global safety. According to this, it is possible at a level of urban transport planning, implementing traffic calming zones, e.g. zone 30, as the Road Code provides. These zones represent, as the French experiences suggest with reference to the fulfilment of analogous measures [12], a suitable tool to establish a link between the speed limit and the road geometric design in which the afore-mentioned limit is in force. The synoptic framework depicted in Figure 3 represents in a schematic way the articulation through phases (pre-analysis, analysis, synthesis) of the methodological path that brings to the identification of routes and/or areas that are open to be treated by traffic calming measures.

\section{Conclusions}

It is a common opinion that implementation of area-wide traffic calming measures has positive safety effects, denoted by reduction of injury or fatal accidents (and injured people); it's also widely held that an overall accident reduction can be a realistic objective as consequence of an urban network management directed towards traffic undesirable effect control.

In the paper it has been shown as such dominant opinions (sometimes similar to undiscerning beliefs) can be very far from the facts when they are based on results from studies lacking in scientific methodological approaches. On the other hand it has been highlighted that in absence of sound studies the statistical approach by means of meta-analysis is able to produce more realistic evaluations (closer to the true effect) of safety benefits of traffic calming measures. Apart from safety aspects of the matter, the reasons of integrating traffic calming measures in the network planning process have been underlined with regards to the area size and the network structure effects. Finally, according to sound international experiences, the methodological path that brings to the identification of routes and/or areas that are promising to be treated by traffic calming measures has been depicted. 


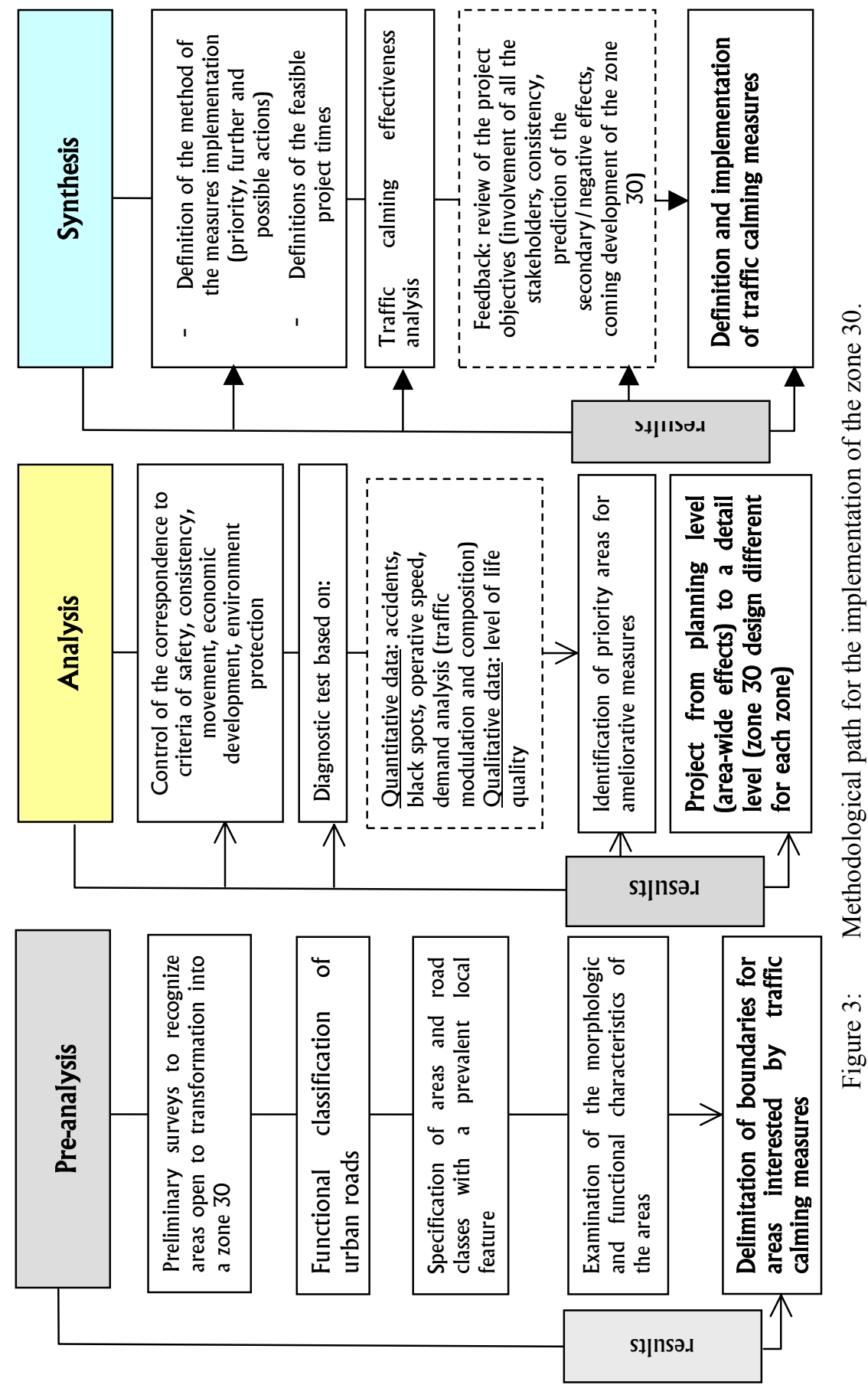




\section{References}

[1] Wilde G.J.S., Can traffic calming devices be expected to reduce the accident rate per head of population or per unit distance driven? Traffic Safety Summit '98, Kananaskis, Alberta, Canada, Oct. 4-7, 1998.

[2] Elvik, R., Mysen, A.B. \& Vaa, T., Trafikksikkerhetshandbok (Traffic Safety Handbook, in Norwegian). Tredje utgave. Oslo, Transportøkonomisk institutt, 1997.

[3] Elvik R., Area-wide urban traffic calming schemes: a meta-analysis of safety effects. Accident Analysis and Prevention, Vol. 33, pp. 327-336, 2001.

[4] Taylor M.C., Lynam D.A. \& Baruya A., The effect of drivers' speed on the frequency of road accidents. TRL Report 421. Transport Research Laboratory, Crowthorne, 2000.

[5] Traffic calming schemes. Opportunities and implementation strategies. Report R-03-22. SWOV Institute for Road Safety Research, Ingrid van Schagen (ed.), Leidschendam, the Netherlands, 2003.

[6] Hummel T., Mackie A. \& Wells P., Traffic calming measures in built-up areas. Literature Review. Unpublished Project Report PR/SE/622/02, TRL, November 2002.

[7] Improving pedestrian safety at unsignalized crossings. TCRP REPORT 112/NCHRP REPORT 562, TRB, Washington DC, 2006.

[8] Hauer E., Observational Before-After Studies in Road Safety. Estimating the Effect of Highway and Traffic Engineering Measures on Road Safety. Pergamon Press, Oxford, 1997.

[9] Borenstein M., Hedges L.\& Rothstein H., (eds). Meta-Analysis Fixed effect vs. random effects. Online www.Meta-Analysis.com, 2007.

[10] Bunn F., Collier T., Frost C., Ker K., Roberts I. \& Wentz R., injuries: systematic review and meta-analysis Traffic calming for the prevention of road traffic. Injury prevention, No. 9, pp. 200-204, doi:10.1136/ip.9.3.200, 2003.

[11] Brilon W \& Blanke H., Area-wide traffic calming measures and their effect on safety in Germany. Conferenza Internazionale vivere e camminare in città, pp. 145-155. Italy, 1994.

[12] Ville plus sure, quartiers sans accidents. Realisations. Evalutations. Certu, Juillet, 1994. 